\title{
Hemangioma de mama simulando metástase no PET-CT*
}

Breast hemangioma mimicking metastasis at PET-CT

\author{
Sabas Carlos Vieira ${ }^{1}$, Jucélia Saraiva e Silva², Eveline Brandão Madeira ${ }^{3}$, Júlio César Queiroz \\ de França ${ }^{3}$, Sebastião Nunes Martins Filho ${ }^{3}$
}

\begin{abstract}
Resumo Hemangioma de mama é um tumor benigno raro que apresenta pouca ou nenhuma captação de ${ }^{18} \mathrm{~F}$-flúor-2-deoxi-Dglicose (FDG) na tomografia por emissão de pósitrons (PET). Relatamos um nódulo mamário compatível, patologicamente, com hemangioma, em uma mulher cuja PET scan demonstrou captação elevada de FDG (simulando tumor maligno). Também fizemos breve revisão das causas que levam a resultados falso-positivos e falso-negativos pela PET. Unitermos: PET/CT; FDG- ${ }^{18}$ F; Hemangioma; Mama.
\end{abstract}

\begin{abstract}
Breast hemangioma is a rare benign tumor that presents either absent or low ${ }^{18} \mathrm{~F}$-fluoro-2-deoxy-D-glucose (FDG) uptake at positron emission tomography (PET). The authors report the case of a breast nodule pathologically compatible with hemangioma in a woman whose PET-scan has demonstrated increased FDG uptake (simulating a malignant tumor). A brief review of factors leading to false positive and false negative PET results is also undertaken.

Keywords: PET/CT; FDG- ${ }^{18} \mathrm{~F}$; Hemangioma; Breast.

Vieira SC, Silva JS, Madeira EB, França JCQ, Martins Filho SN. Hemangioma de mama simulando metástase no PET-CT. Radiol Bras. 2011 Nov/Dez;44(6):401-402.
\end{abstract}

\section{INTRODUÇÃO}

Os hemangiomas são tumores vasculares benignos, raros na mama e com baixa captação de ${ }^{18}$ F-flúor-2-deoxi-D-glicose (FDG) na tomografia por emissão de pósitrons (PET), podendo ser distinguido de tumores malignos ${ }^{(\mathbf{1})}$.

Para distinguir lesões malignas de benignas tem-se utilizado a FDG-PET, que detecta o metabolismo aumentado de glicose, geralmente maior em tumores malignos que em benignos ${ }^{(2)}$. No entanto, não apresenta a mesma precisão anatômica que a tomografia computadorizada (CT), utilizando-se, por isso, uma técnica que combina os dois scanners (PET/CT). Todavia, falsos resultados positivos e negativos foram relatados ${ }^{(3)}$.

\footnotetext{
* Trabalho realizado na Universidade Federal do Piauí (UFPI) Teresina, PI, Brasil.

1. Médico Oncologista e Mastologista, Presidente da Sociedade Brasileira de Mastologia, Professor Titular da Disciplina de Oncologia da Faculdade de Medicina da Universidade Federal do Piauí (UFPI), Teresina, PI, Brasil.

2. Médica Patologista, Médica do Departamento de Patologia e Clínica Médica da Medlmagem, Teresina, PI, Brasil.

3. Acadêmicos de Medicina da Universidade Federal do Piau (UFPI), Teresina, PI, Brasil.

Endereço para correspondência: Eveline Brandão Madeira. Rua Fidalma Martins de Carvalho, 4355, BI-06, ap. 204, Ininga. Teresina, PI, Brasil, 64048-480. E-mail: evelinemadeira@ hotmail.com

Recebido para publicação em 14/1/2011. Aceito, após revisão, em 21/6/2011.
}

Este estudo consiste em relatar um caso de hemangioma de mama em uma mulher cuja PET/CT demonstrou captação elevada de FDG (simulando tumor maligno). Também fizemos uma breve revisão das causas que levam a resultados falso-positivos e falso-negativos pela PET.

\section{RELATO DO CASO}

Paciente do sexo feminino, 63 anos, apresentou, ao exame clínico, nódulo medindo cerca de $1,5 \mathrm{~cm}$ na união dos quadrantes inferiores da mama direita, de contornos parcialmente definidos, com consistência fibroelástica e axila clinicamente negativa. De antecedentes, a paciente apresentava um tumor de colón já operado, e após submeter-se a quimioterapia adjuvante evoluiu com metástase pulmonar.

A mamografia demonstrou uma lesão nodular de contornos lobulados parcialmente delimitados na mama direita e a ultrassonografia revelou tratar-se de lesão sólida medindo $1,8 \times 0,7 \mathrm{~cm}$, isoecogênica em relação ao tecido mamário, classificada como BI-RADS 4.

Foi realizada FDG-PET/CT, que revelou aumento da captação de FDG no nódulo mamário (Figura 1). Com este achado, foi indicada ressecção do nódulo. O histopa- tológico final revelou tratar-se de neoplasia vascular benigna medindo 1,6 × 1,5 $\times$ $0,5 \mathrm{~cm}$, constituída por vasos sanguíneos de paredes delgadas, ectasiados e congestos inseridos no estroma conjuntivo, além de ausência de atipias, compatível com hemangioma capilar.

\section{DISCUSSÃO}

Os hemangiomas capilares são tumores vasculares benignos caracterizados por proliferação de vasos capilares ${ }^{(4)}$. $\mathrm{O}$ hemangioma da mama afeta principalmente mulheres pós-menopáusicas e pode aumentar de tamanho na reposição hormonal ${ }^{(\mathbf{5})}$.

Há vários métodos de imagem para a detecção, diagnóstico e conduta das doenças mamárias, mas a mamografia continua sendo a mais importante técnica de imagem para as mamas. Para promover uma uniformização dos achados mamográficos, foi desenvolvida a classificação BI-RADS, subdividindo-os em cinco classes: negativo, benigno, provavelmente benigno, suspeito e altamente suspeito ${ }^{(6)}$.

Alguns estudos relatam que o hemangioma mamário se apresenta à mamografia como uma lesão macrolobular bem circunscrita que pode conter calcificação ${ }^{(7,8)}$. No entanto, estes achados não são especí- 

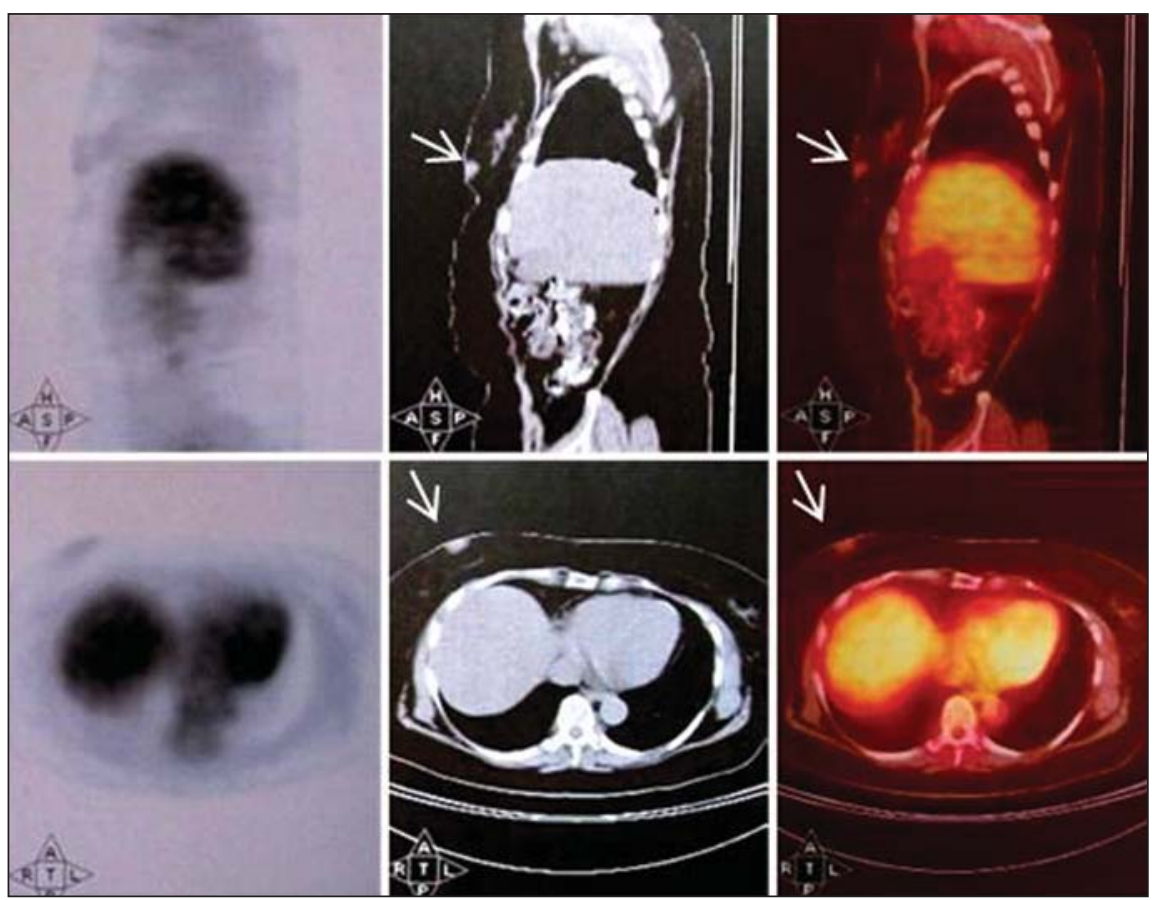

Figura 1. Tomografia por emissão de pósitrons acoplada a tomografia computadorizada (FDG-PET/TC) revelando aumento da captação de FDG na mama (setas).

ficos, o que pode explicar o número significativo de hemangiomas classificados como BI-RADS 3 ou 4 e a não diferenciação com fibroadenomas ou $\operatorname{cistos}^{(8)}$.

A PET/CT capta a atividade hiperglicolítica de células malignas pelo acúmulo da FDG, que é um análogo da glicose. Seu acúmulo ocorre por um aumento do número de proteínas transportadoras de glicose e dos níveis de hexoquinase e fosfofrutoquinase, que promovem a glicólise ${ }^{(\mathbf{3})}$. Depois de fosforilado à FDG-6-fosfato pela hexoquinase, não pode mais ser metabolizado ou transportado para o espaço extracelular, sendo acumulado seletivamente nas células tumorais ${ }^{(2)}$.

Resultados falso-negativos podem ser encontrados na PET scan em tumores com baixa atividade glicolítica, tais como adenomas, linfomas de baixo grau e nos de pequeno tamanho ${ }^{(9)}$. Além disso, em doenças localizadas próximas aos locais de captação fisiológica (coração, rins, bexiga e fígado), a FDG-PET deve ser complemen- tada com outras modalidades de imagem para confirmar os resultados ${ }^{(3)}$. A realização de quimioterapia dentro de um mês de um exame FDG-PET pode diminuir a sua sensibilidade, em razão do reduzido número de células de tumor metabolicamente ativo, o que nem sempre prediz uma boa resposta $^{(2)}$.

Por outro lado, resultados falso-positivos podem ocorrer em doenças infecciosas e inflamatórias nas quais macrófagos ativados e neutrófilos mostram aumento da acumulação de FDG por utilizarem glicose como fonte de energia para a quimiotaxia e fagocitose ${ }^{(\mathbf{1 0})}$. Tem-se sugerido que as células inflamatórias utilizam mais glicose sob hiperglicemia do que em euglicemia e, portanto, lesões com tais células são mais interpretadas como lesões malignas em hiperglicemia $^{(3)}$.

O acúmulo de FDG em hemangioma pode estar relacionado à retenção de sangue na lesão, que resulta em isquemia localizada. A hipóxia resultante pode então acelerar a glicólise anaeróbia, que leva a alta absorção de FDG $^{(\mathbf{1 1})}$.

\section{CONCLUSÃO}

Apesar de ser um exame com significativa contribuição para o diagnóstico, tratamento e avaliação de pacientes oncológicos, a FDG-PET/CT pode gerar resultados falso-positivos ou falso-negativos. Dessa forma, a compreensão das causas de falsos resultados é essencial para evitar diagnósticos equivocados.

\section{REFERÊNCIAS}

1. Sakurai K, Haram M, Ozawa Y, et al. Thoracic hemangiomas: imaging via $\mathrm{CT}, \mathrm{MR}$, and PET along with pathologic correlation. J Thorac Imaging. 2008;23:114-20.

2. Selzner M, Hany TF, Wildbrett P, et al. Does the novel PET/CT imaging modality impact on the treatment of patients with metastatic colorectal cancer of the liver? Ann Surg. 2004;240:102734; discussion 1035-6.

3. Chang JM, Lee HJ, Goo JM, et al. False positive and false negative FDG-PET scans in various thoracic diseases. Korean J Radiol. 2006; 7:5769

4. Courcoutsakis NA, Hill SC, Chow CK, et al. Breast hemangiomas in a patient with KasabachMerritt syndrome: imaging findings. AJR Am J Roentgenol. 1997;169:1397-9.

5. Mariscal A, Casas JD, Balliu E, et al. Breast hemangioma mimicking carcinoma. Breast. 2002; $11: 357-8$

6. Vieira AV, Toigo FT. Classificação BI-RADS categorização de 4.968 mamografias. Radiol Bras. 2002;35:205-8.

7. Siewert B, Jacobs T, Baum JK. Sonographic evaluation of subcutaneous hemangioma of the breast. AJR Am J Roentgenol. 2002;178:1025-7.

8. Mesurolle B, Sygal V, Lalonde L, et al. Sonographic and mammographic appearances of breast hemangioma. AJR Am J Roentgenol. 2008;191: W17-22.

9. Carter KR, Kotlyarov E. Common causes of false positive $\mathrm{F}^{18}$ FDG PET/CT scans in oncology. Braz Arch Biol Technol. 2007;50(special number):29_ 35.

10. Shim SS, Lee KS, Kim BT, et al. Focal parenchymal lung lesions showing a potential of falsepositive and false-negative interpretations on integrated PET/CT. AJR Am J Roentgenol. 2006; 186:639-48.

11. Hatayama K, Watanabe H, Ahmed AR, et al Evaluation of hemangioma by positron emission tomography: role in a multimodality approach. $\mathrm{J}$ Comput Assist Tomogr. 2003;27:70-7. 